\title{
P171
}

\section{Grapevine downy mildew dual epidemics: a leaf and inflorescence transcriptomics study}

\author{
Daniele Buonassisi $^{(1)}$ - Michele Perazzolli(2) - Elisa Peressotti ${ }^{(2)}$ - Alice Tadiello(2) - Rita Musetti( $^{(3)}$ - \\ Riccardo Velasco(2) - Dario Cantu(4) - Silvia Vezzulli ${ }^{(2)}$

\begin{abstract}
Fondazione Edmund Mach, Research and Innovation Centre, Università degli Studi di Udine, Department of Enology, Davis, United States ${ }^{(4)}$
\end{abstract} \\ Agricultural and Environmental Sciences, San Michele all'Adige (TN)/Udine, Italy ${ }^{(1)}$ - Fondazione Edmund Mach, \\ Research and Innovation Centre, San Michele all'Adige (TN), Italy ${ }^{(2)}$ - Università degli Studi di Udine, Department \\ of Agricultural and Environmental Sciences, Udine, Italy ${ }^{(3)}$ - University of California, Department of Viticulture and
}

Grapevine downy mildew (DM), caused by the oomycete Plasmopara viticola (Berk. \& Curt.) Berl. \& de Toni, is one of the most important diseases affecting viticulture, especially in humid climates. DM is controlled with the use of fungicides with considerable economic costs as well as negative impact on the environment, human health and public perception.

Dual epidemics are defined as infections developing on two or more plant organs in the course of a cropping season. Dual epidemics are particularly important when one of the organs affected by the disease constitutes the valuable part of the plant. $P$. viticola can infect every green tissue of the grapevine, including flowers and berries. Different organs of the same genotype can vary in their susceptibility to DM. The organ-specific nature of DM response makes difficult to extrapolate resistance from leaf to fruit and vice versa.

The objective of this study was to investigate the differences of gene expression between grapevine organs, which may underlie the organ-specific susceptibility to DM. Based on a previous phenotypic screening under field and controlled conditions, we selected Cabernet Cortis and its parent Cabernet Sauvignon, which display different susceptibility to DM. The former has mid-resistant leaves and mid-susceptible inflorescences to DM, while the latter has both mid-susceptible leaves and inflorescences to DM. To profile their genome-wide transcriptional responses to DM, we constructed twenty-four RNA-seq libraries using leaves and inflorescences (E-L 15/17) derived from fruiting cuttings grown under controlled conditions infected by $P$. viticola. Mock-inoculated samples were included as controls. Bioinformatics analyses are in progress to identify differentially expressed genes between organs within the same genotype and between the two genotypes.

\section{P172}

\section{GLRaV-3 full-length and mini-replicon: new prospects for Grapevine Leafroll Disease and functional genomics}

\author{
Johan Burger $^{(1)}$ \\ Department of Genetics, Stellenbosch University, Stellenbosch, South Africa ${ }^{(1)}$
}

Grapevine Leafroll Disease (GLD) is one of the most destructive virus diseases affecting grapevine globally. Among the several viruses associated with GLD, Grapevine leafroll-associated virus 3 (GLRaV-3) belonging to the genus Ampelovirus, and family Closteroviridae, is by far the most prevalent and has a world-wide distribution. The single-strand RNA genome has been sequenced and most of the proteins, which it encodes, identified by sequence similarity. However, for most of these, the specific function is still unknown. While 


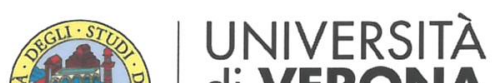
di VERONA

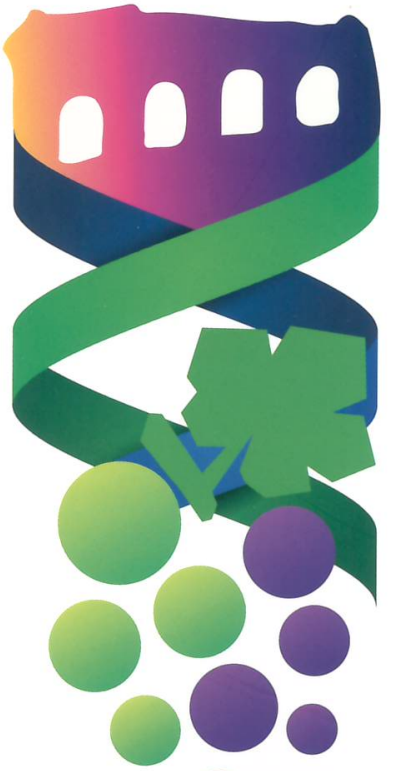

X INTERNATIONAL SYMPOSIUM ON GRAPEVINE PHYSIOLOGY AND BIOTECHNOLOGY

政

\title{
5
}
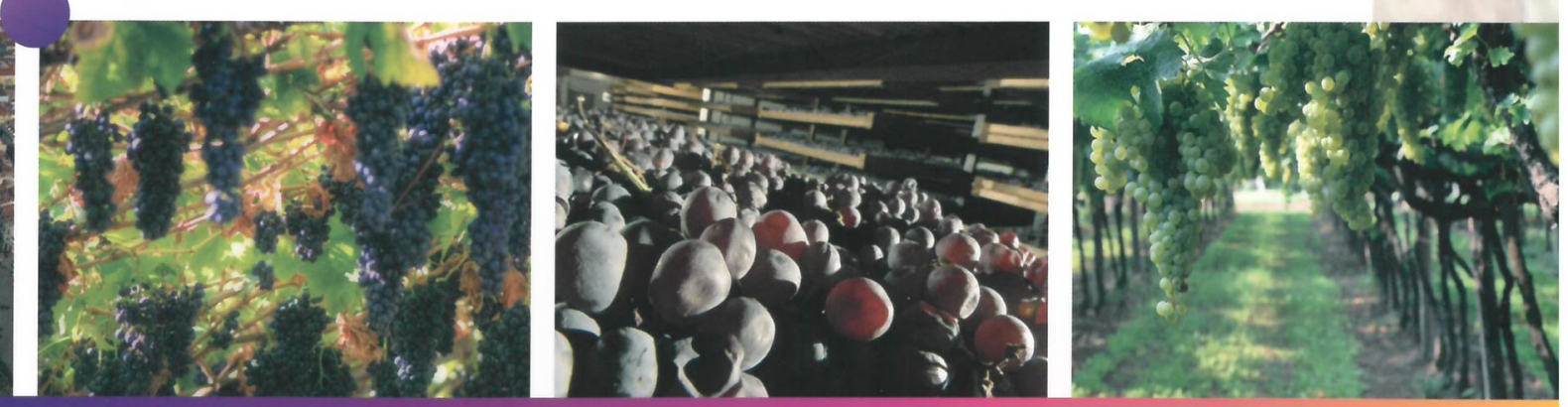

BOOK OF ABSTRACTS

\author{
June $13^{\text {th }}-18^{\text {th }}, 2016$ \\ Palazzo della Gran Guardia \\ Verona, Italy
}

